\title{
Eulerian finite element method for parabolic PDEs on implicit surfaces
}

\author{
G. DZIUK ${ }^{\dagger}$ \\ Abteilung für Angewandte Mathematik, University of Freiburg, \\ Hermann-Herder-Straße 10, D-79104 Freiburg i. Br., Germany \\ C. M. ELLIOTT \\ Mathematics Institute, University of Warwick, Coventry CV4 7AL, United Kingdom
}

[Received 25 October 2006 and in revised form 8 November 2007]

\begin{abstract}
We define an Eulerian level set method for parabolic partial differential equations on a stationary hypersurface $\Gamma$ contained in a domain $\Omega \subset \mathbb{R}^{n+1}$. The method is based on formulating the partial differential equations on all level surfaces of a prescribed function $\Phi$ whose zero level set is $\Gamma$. Eulerian surface gradients are formulated by using a projection of the gradient in $\mathbb{R}^{n+1}$ onto the level surfaces of $\Phi$. These Eulerian surface gradients are used to define weak forms of surface elliptic operators and so generate weak formulations of surface elliptic and parabolic equations. The resulting equation is then solved in one dimension higher but can be solved on a mesh which is unaligned to the level sets of $\Phi$. We consider both second order and fourth order elliptic operators with natural second order splittings. The finite element method is applied to the weak form of the split system of second order equations using piecewise linear elements on a fixed grid. The computation of the mass and element stiffness matrices is simple and straightforward. Numerical experiments are described which indicate the power of the method. We describe how this framework may be employed in applications.
\end{abstract}

\section{Introduction}

There has been burgeoning interest in the computation of partial differential equations on curves and surfaces. Models involving partial differential equations on surfaces arise in many areas including material science, bio-physics, fluid mechanics and image processing. For example, we refer to [10, 27. 29] for applications of the Allen-Cahn and Cahn-Hilliard equations to phase ordering and separation on surfaces. Models for thin fluid films on surfaces have been developed in [21, 24]. For image processing and geometry applications we mention geodesic flow of curves on surfaces and active contours for segmentation on surfaces, [6, 22, 23, 28].

The work in this paper is concerned with an approach to the formulation and approximation of parabolic equations on a prescribed stationary $n$-dimensional surface $\Gamma$ in $\mathbb{R}^{n+1}(n=1,2)$ using an implicit representation of the surface. The surface is just one level set of a prescribed function $\Phi$ and the partial differential equation and its solution are extended to a domain $\Omega \subset$ $\mathbb{R}^{n+1}$ containing the surface. A general framework for formulating partial differential equations on implicit surfaces was proposed by the authors of [3]. They considered time dependent second order linear and nonlinear diffusion equations in the context of finite difference approximations on rectangular grids independent of the surfaces. In [20, 19] the authors presented finite difference

\footnotetext{
${ }^{\dagger}$ E-mail: gerd.dziuk@mathematik.uni-freiburg.de

${ }^{\ddagger}$ E-mail: C.M.Elliott@ warwick.ac.uk
} 
methods for fourth order parabolic equations on implicit surfaces. A finite element approximation of elliptic equations on implicit surfaces is presented in [5].

Our work is concerned with the finite element discretization of second and fourth order parabolic equations on surfaces. The idea is to solve PDEs on all level surfaces of $\Phi$ in $\Omega$ by discretizing a suitable variational formulation by a finite element method on a mesh which is independent of the surfaces. This defines an Eulerian formulation. Stable time stepping schemes are formulated in a natural way. By using second order splitting of the fourth order operators, $H^{1}$ conforming finite element schemes can be employed for fourth order problems such as the Cahn-Hilliard equation. When the boundary of $\Omega$ consists of level sets of $\Phi$ it is not necessary to impose artificial boundary conditions because the triangulation is fitted to the domain $\Omega$. A remarkable feature of our numerical experiments is that, on a fixed level set, finite element approximations converge at an optimal rate. Our approach can be extended to second order diffusion problems on evolving surfaces (see [14]). See also [1] and [30]. The computing times for our method are similar to computing times for cartesian PDEs.

This approach is in contrast to approximating the PDEs directly on triangulated surfaces. In [11], [12] and [13] we introduced the surface and evolving surface finite element method (respectively SFEM and ESFEM) for the numerical solution of elliptic and parabolic equations on prescribed stationary and moving hypersurfaces. The method relies on approximating the partial differential equation on a triangulated surface $(n=2)$ or polygonal curve $(n=1)$. Naturally, where applicable, this method is more efficient than solving PDEs on implicit surfaces. On the other hand, in applications a surface might arise as a level set of a function computed from solving another coupled equation in which case the method of this paper may be attractive. Also when the surface is complex and evolving with possible topology changes it may be advantageous to employ a level set description of the surface. Finally, the method is appropriate when a PDE has to be solved on all level sets of a given function.

The layout of the paper is as follows. We begin in Section 2 by defining notation and essential concepts from elementary differential geometry necessary to describe the problem and the numerical method. The equations and variational formulations are presented in Section 3 In Section 4 the finite element method is defined. The results of numerical experiments are presented in Section 5 . Finally, in Section 6 we make some concluding remarks.

\section{Level set tangential gradients}

Let $\Gamma$ be a compact Lipschitz hypersurface without boundary in $\mathbb{R}^{n+1}$ which has a representation defined by a level set function $\Phi=\Phi(x), x \in \mathbb{R}^{n+1}$, so that

$$
\Gamma=\{x \in \bar{\Omega}: \Phi(x)=0\}
$$

where $\Omega$ is an open subset of $\mathbb{R}^{n+1}$ with Lipschitz boundary $\partial \Omega$ and unit outward pointing normal $v_{\partial \Omega}$. We assume that $\Phi$ satisfies the nondegeneracy condition

$$
\nabla \Phi \neq 0 \quad \text { in } \bar{\Omega} .
$$

In particular we suppose that

$$
\Phi \in C^{0,1}(\bar{\Omega}) .
$$


The orientation of $\Gamma$ is set by taking the normal $v$ to $\Gamma$ to be in the direction of increasing $\Phi$. Then we define an extension of $v$ to all of $\Omega$ using the normal vector field

$$
v(x)=\frac{\nabla \Phi(x)}{|\nabla \Phi(x)|}, \quad x \text { in } \bar{\Omega} .
$$

A possible choice for $\Phi$ is a signed distance function $d$ and in that case $|\nabla \Phi|=|\nabla d|=1$ on $\Omega$.

We define the projection

$$
\mathcal{P}_{\Phi}:=I-v \otimes v, \quad\left(\mathcal{P}_{\Phi}\right)_{i j}=\delta_{i j}-v_{i} v_{j}, \quad i, j=1, \ldots, n+1 .
$$

Thus $\mathcal{P}(x)$ is the projection onto the tangent space of the surface $\Gamma_{r}:=\left\{y \in \mathbb{R}^{n+1}: \Phi(y)=r\right\}$, $r=\Phi(x)$, so that $\mathcal{P}_{\Phi} v=0$. We define the Eulerian surface gradient by

$$
\nabla_{\Phi} \eta:=\mathcal{P}_{\Phi} \nabla \eta
$$

and observe that

$$
\nabla_{\Phi} \eta=\nabla \eta-\nabla \eta \cdot v v
$$

where, for $x$ and $y$ in $\mathbb{R}^{n+1}, x \cdot y$ is the Euclidean scalar product and $\nabla \eta$ denotes the usual gradient on $\mathbb{R}^{n+1}$. Note that

$$
\nabla_{\Phi} \eta \cdot v=0
$$

and that for any level surface $\Gamma_{r}$,

$$
\nabla_{\Gamma_{r}} \eta:=\left.\nabla_{\Phi} \eta\right|_{\Gamma_{r}}
$$

only depends on the values of $\eta$ restricted to $\Gamma_{r}$ and is the tangential (surface) gradient on $\Gamma_{r}$. Denoting the components of $\nabla_{\Phi} \eta$ by $D_{i}^{\Phi} \eta:=\partial_{x_{i}} \eta-\nabla \eta \cdot v v_{i}$ we define the surface divergence of a vector field $\xi$ by

$$
\nabla_{\Phi} \cdot \xi=\sum_{i=1}^{n+1} D_{i}^{\Phi} \xi_{i}
$$

Eulerian surface elliptic operators can then be defined in a natural way. For example the Eulerian Laplace-Beltrami operator is defined by

$$
\Delta_{\Phi} \eta=\nabla_{\Phi} \cdot \nabla_{\Phi} \eta .
$$

The Eulerian mean curvature of a level surface of $\Phi \in C^{1,1}(\bar{\Omega})$ is defined by

$$
H_{\Phi}:=-\nabla \cdot v=-\nabla \cdot \frac{\nabla \Phi}{|\nabla \Phi|} .
$$

We recall the coarea formula:

LEMMA 2.1 (Coarea formula) Let $\Phi: \Omega \rightarrow \mathbb{R}$ be Lipschitz continuous with $\Phi_{M}:=\sup \Phi$ and $\Phi_{m}=\inf \Phi$. Assume that for for each $r \in \mathbb{R}$ the level set $\Gamma_{r}:=\left\{x \in \mathbb{R}^{n+1}: \Phi(x)=r\right\}$ is a Lipschitz $n$-dimensional hypersurface in $\mathbb{R}^{n+1}$. Suppose $\eta: \Omega \rightarrow \mathbb{R}$ is continuous and integrable. Then

$$
\int_{\Phi_{m}}^{\Phi_{M}}\left(\int_{\Gamma_{r}} \eta\right) \mathrm{d} r=\int_{\Omega} \eta|\nabla \Phi|
$$


For $\Phi \in C^{1,1}(\bar{\Omega})$ we define the Hilbert spaces

$$
\begin{aligned}
& L_{\Phi}^{2}(\Omega):=\left\{\eta \text { Lebesgue measurable on } \Omega:\langle\eta, \eta\rangle_{\Phi}<\infty\right\}, \\
& H_{\Phi}^{1}(\Omega):=\left\{\eta \in L_{\Phi}^{2}(\Omega): \nabla_{\Phi} \eta \in L_{\Phi}^{2}(\Omega)\right\}
\end{aligned}
$$

where

$$
\langle\eta, \eta\rangle_{\Phi}:=\int_{\Omega} \eta^{2}|\nabla \Phi|, \quad\langle\eta, \eta\rangle_{H_{\Phi}^{1}}:=\langle\eta, \eta\rangle_{\Phi}+\left\langle\nabla_{\Phi} \eta, \nabla_{\Phi} \eta\right\rangle_{\Phi}
$$

and we set

$$
\|\eta\|_{L_{\Phi}^{2}(\Omega)}:=\langle\eta, \eta\rangle_{\Phi}^{1 / 2}, \quad\|\eta\|_{H_{\Phi}^{1}(\Omega)}:=\left\{\|\eta\|_{L_{\Phi}^{2}(\Omega)}^{2}+\left\|\nabla_{\Phi} \eta\right\|_{L_{\Phi}^{2}(\Omega)}^{2}\right\}^{1 / 2} .
$$

Here we use the distributional derivative $\nabla_{\Phi} \eta$ which is defined in the usual way using the equation (2.9) of the following lemma which gives the Eulerian formula for integration by parts over level surfaces.

LEMMA 2.2 (Eulerian integration by parts) Let $\eta \in H_{\Phi}^{1}(\Omega)$ and $\mathcal{Q} \in\left(H_{\Phi}^{1}(\Omega)\right)^{n+1}$. Then

$$
\begin{aligned}
& \int_{\Omega} \nabla_{\Phi} \eta|\nabla \Phi|=-\int_{\Omega} \eta H_{\Phi} v|\nabla \Phi|+\int_{\partial \Omega} \eta\left(v_{\partial \Omega}-v \cdot v_{\partial \Omega} v\right)|\nabla \Phi|, \\
& \int_{\Omega} \nabla_{\Phi} \cdot(\eta \mathcal{Q})|\nabla \Phi|=-\int_{\Omega} H_{\Phi} \eta \mathcal{Q} \cdot v|\nabla \Phi|+\int_{\partial \Omega} \eta \mathcal{Q} \cdot\left(v_{\partial \Omega}-v \cdot v_{\partial \Omega} v\right)|\nabla \Phi| .
\end{aligned}
$$

Proof. We prove the first equation, from which the second follows. We employ the notation $\partial_{i}:=$ $\partial / \partial x_{i}$ and $\partial_{i j}:=\partial^{2} / \partial x_{i} \partial x_{j}$. Elementary calculations yield

$$
\begin{aligned}
\partial_{i}|\nabla \Phi| & =v_{k} \partial_{i k} \Phi=\left(D^{2} \Phi v\right)_{i}, \\
|\nabla \Phi| \partial_{j} v_{k} & =\partial_{j k} \Phi-v_{k}\left(D^{2} \Phi v\right)_{j}, \\
|\nabla \Phi| H_{\Phi} & =-\operatorname{Tr}\left(D^{2} \Phi\right)+v \cdot D^{2} \Phi v,
\end{aligned}
$$

where $D^{2} \Phi$ is the Hessian matrix of second derivatives, $\operatorname{Tr}(\cdot)$ is the trace of a matrix and we employ the summation convention for repeated indices.

Using the definition of $\nabla_{\Phi}$ we find that

$$
\text { LHS }:=\int_{\Omega} \nabla_{\Phi} \eta|\nabla \Phi|=\int_{\Omega}|\nabla \Phi|(\nabla \eta-v \cdot \nabla \eta v)
$$

and then we employ the standard integration formula on $\Omega$. It follows that

$(\mathrm{LHS})_{i}=-\int_{\Omega} \eta \partial_{i}|\nabla \Phi|+\int_{\Omega} \eta \partial_{m}\left(v_{i} v_{m}|\nabla \Phi|\right)+\int_{\partial \Omega} \eta|\nabla \Phi|\left(\left(v_{\partial \Omega}\right)_{i}-v_{i}\left(\nu \cdot v_{\partial \Omega}\right)\right)=I+I I+I I I$.

Straightforward calculations yield

$$
I I=\int_{\Omega} \eta\left(v_{i} \operatorname{Tr}\left(D^{2} \Phi\right)+\left(D^{2} \Phi v\right)_{i}-v \cdot D^{2} \Phi v v_{i}\right) .
$$

Combining $I$ and $I I$ using the formula for $H_{\Phi}$ gives the desired result.

REMARK 2.3 The boundary terms in the integration by parts formulae disappear when $v=v_{\partial \Omega}$. 


\section{PDEs on surfaces}

\subsection{Conservation and diffusion}

Let $\Phi: \Omega \rightarrow \mathbb{R}$ be a prescribed nondegenerate level set function. Let $\mathcal{Q}: \Omega \rightarrow \mathbb{R}^{n+1}$ be a given flux. Then the Eulerian conservation law we consider is

$$
\frac{\mathrm{d}}{\mathrm{d} t} \int_{R}|\nabla \Phi| u=-\int_{\partial R} \mathcal{Q} \cdot v_{\partial R}
$$

for each subdomain $R$ of $\Omega$ where $v_{R}$ is the outward unit normal to $\partial R$. In particular we consider a flux of the form

$$
\mathcal{Q}=|\nabla \Phi| q_{\Phi}
$$

where $q_{\Phi}: \Omega \rightarrow \mathbb{R}^{n+1}$ is a flux satisfying

$$
q_{\Phi} \cdot v=0
$$

Since

$$
\frac{\mathrm{d}}{\mathrm{d} t} \int_{R}|\nabla \Phi| u=\int_{R} u_{t}|\nabla \Phi|
$$

and

$$
\int_{\partial R} q_{\Phi} \cdot v_{\partial R}|\nabla \Phi|=\int_{R} \nabla_{\Phi} \cdot q_{\Phi}|\nabla \Phi|,
$$

where we have used Eulerian integration by parts 2.10) and 3.2, it follows that

$$
\int_{R}|\nabla \Phi|\left(u_{t}+\nabla_{\Phi} \cdot q_{\Phi}\right)=0
$$

for every subdomain $R$, which implies the partial differential equation

$$
u_{t}+\nabla_{\Phi} \cdot q_{\Phi}=0 \quad \text { in } \Omega .
$$

We take for the constitutive law that $q_{\Phi}$ is a diffusive flux given by

$$
q_{\Phi}=-\mathcal{D} \nabla_{\Phi} w
$$

Here $w$ is another field variable which will be defined in terms of $u$ by a constitutive relation and $\mathcal{D}$ is a symmetric diffusion tensor with the property

$$
\mathcal{D} v^{\perp} \cdot v=0
$$

for every tangent vector $v^{\perp}$. We assume that there exists a $d_{0}>0$ such that

$$
z \cdot \mathcal{D} z \geqslant d_{0} z \cdot z \quad \text { for all } z \cdot v=0
$$

This leads to the diffusion equation

$$
u_{t}-\nabla_{\Phi} \cdot\left(\mathcal{D} \nabla_{\Phi} w\right)=0 \quad \text { on } \Omega \text {. }
$$


Throughout we assume the initial condition

$$
u(\cdot, 0)=u_{0}(\cdot)
$$

The constitutive relation between $u$ and $w$ is still to be defined. Observe that 3.8 can be written as

$$
u_{t}=\mathcal{P}_{\Phi} \nabla \cdot\left(\mathcal{D} \mathcal{P}_{\Phi} \nabla w\right),
$$

which can be seen as a degenerate parabolic equation, depending on the relation between $w$ and $u$, because $\mathcal{P}_{\Phi}$ has a zero eigenvalue in the normal direction $v$.

The variational form is then obtained in the following way. We multiply equation $(3.8)$ by a test function $\eta$ and integrate to obtain

$$
\int_{\Omega}\left\{u_{t}-\nabla_{\Phi} \cdot\left(\mathcal{D} \nabla_{\Phi} w\right)\right\} \eta|\nabla \Phi|=0 .
$$

Observe that integration by parts, 2.10 , together with the observation that

$$
\mathcal{D} \nabla_{\Phi} w \cdot v=0
$$

gives

$$
\int_{\Omega} \mathcal{D} \nabla_{\Phi} w \cdot \nabla_{\Phi} \eta|\nabla \Phi|=-\int_{\Omega} \nabla_{\Phi} \cdot \mathcal{D} \nabla_{\Phi} w \eta|\nabla \Phi|+\int_{\partial \Omega} \mathcal{D} \nabla_{\Phi} w \cdot v_{\partial \Omega} \eta|\nabla \Phi| .
$$

In order to proceed we need a boundary condition for $w$ on $\partial \Omega$. It is natural to impose the zero flux condition

$$
|\nabla \Phi| \mathcal{D} \nabla_{\Phi} w \cdot v_{\partial \Omega}=0 \quad \text { on } \partial \Omega
$$

and obtain the equivalent variational equation

$$
\int_{\Omega} u_{t} \eta|\nabla \Phi|+\int_{\Omega} \mathcal{D} \nabla_{\Phi} w \cdot \nabla_{\Phi} \eta|\nabla \Phi|=0
$$

REMARK 3.1 (Conservation) Let $\xi: \mathbb{R} \rightarrow \mathbb{R}$ be an arbitrary smooth function and set $\eta=\xi(\Phi)$. Since $\nabla_{\Phi} \eta=\xi^{\prime}(\Phi) \nabla_{\Phi} \Phi=0$ we find the conservation equation

$$
\frac{\mathrm{d}}{\mathrm{d} t} \int_{\Omega} u \xi(\Phi)|\nabla \Phi|=0
$$

It follows from the coarea formula that

$$
\int_{\Phi_{m}}^{\Phi_{M}} \xi(r)\left(\int_{\Gamma_{r}} u\right) \mathrm{d} r=\int_{\Phi_{m}}^{\Phi_{M}} \xi(r)\left(\int_{\Gamma_{r}} u_{0}\right) \mathrm{d} r
$$

which implies that on each level surface $\Gamma_{r}=\{x: \Phi(x)=r\}$ of $\Phi$ we have conservation

$$
\int_{\Gamma_{r}} u=\int_{\Gamma_{r}} u_{0} .
$$


EXAMPLE 3.2 (Linear diffusion) Setting $w=u$ and $\mathcal{D}=\mathcal{I}$ we find the heat equation on surfaces,

$$
u_{t}=\Delta_{\Phi} u,
$$

and 3.11 becomes

$$
\frac{\mathrm{d}}{\mathrm{d} t} \int_{\Omega} u \eta|\nabla \Phi|+\int_{\Omega} \nabla_{\Phi} u \cdot \nabla_{\Phi} \eta|\nabla \Phi|=0 .
$$

Note that for smooth $g: \mathbb{R} \rightarrow \mathbb{R}$ the function $u=g(\Phi)$ is $\Phi$-harmonic, that is,

$$
\Delta_{\Phi} u=0 .
$$

Setting

$$
g(r)=\frac{1}{\left|\Gamma_{r}\right|} \int_{\Gamma_{r}} u_{0}
$$

we see that in the case of no-flux boundary conditions the long time steady state solution is

$$
u_{\infty}=g(\Phi) .
$$

However, as we will see in the computational Example 5.7 in Section 5, it is possible for $g$ defined by (3.17) to be discontinuous, which leads to discontinuous $\Phi$-harmonic functions.

Furthermore, it is interesting to observe that the unique steady state of the equation under no-flux boundary conditions for any positive $\lambda$,

$$
u_{t}=\Delta_{\Phi} u-\lambda(u-\Phi),
$$

is $u_{\infty}=\Phi$.

REMARK 3.3 (Diffusion in a layered medium) Observing that

$$
\nabla_{\Phi} \cdot \tau=\frac{1}{|\nabla \Phi|} \nabla \cdot(|\nabla \Phi| \tau), \quad \forall \tau \cdot \nu=0,
$$

we can rewrite the diffusion equation 3.8 as

$$
(|\nabla \Phi| u)_{t}=\nabla \cdot\left(\mathcal{D}_{\Phi} \nabla w\right)
$$

where

$$
\mathcal{D}_{\Phi}=|\nabla \Phi| \mathcal{D} \mathcal{P}_{\Phi} .
$$

Thus we may view 3.8 as a usual diffusion equation in $\mathbb{R}^{n+1}$ with a special diffusivity tensor and mass density. We interpret this as a diffusion equation for a striated or layered material whose layers are infinitesimally thick, tangential to the level surfaces of $\Phi$ and are insulated from each other.

EXAMPLE 3.4 (Fourth order linear diffusion) Setting

$$
w=-\Delta_{\Phi} u
$$

leads to the fourth order linear diffusion equation

$$
u_{t}=-\nabla_{\Phi} \cdot \mathcal{D} \nabla_{\Phi}\left(\Delta_{\Phi} u\right) .
$$


Using the boundary condition

$$
\nabla_{\Phi} u \cdot v_{\partial \Omega}=0
$$

and splitting into two second order equations we obtain the following definition of a weak solution which only uses $H^{1}$ spaces: The pair $(u, w) \in\left(H_{\Phi}^{1}(\Omega)\right)^{2}$ is a weak solution of 3.23 if

$$
\begin{array}{r}
\int_{\Omega} u_{t} \eta|\nabla \Phi|+\int_{\Omega} \mathcal{D} \nabla_{\Phi} w \cdot \nabla_{\Phi} \eta|\nabla \Phi|=0, \\
\int_{\Omega} \nabla_{\Phi} u \cdot \nabla_{\Phi} \eta|\nabla \Phi|-\int_{\Omega} w \eta|\nabla \Phi|=0,
\end{array}
$$

for all $\eta \in H_{\Phi}^{1}(\Omega)$.

\subsection{More equations}

Other standard equations can be formulated on implicit surfaces in a straightforward manner.

EXAMPLE 3.5 (Nonlinear diffusion) Setting $w=f(u)$ and $\mathcal{D}=m(u) \mathcal{I}$ we find the nonlinear diffusion equation

$$
u_{t}=\nabla_{\Phi} \cdot\left(K(u) \nabla_{\Phi} u\right)
$$

where $K(u)=m(u) f^{\prime}(u)$. Linear diffusion and the porous medium equation are recovered by suitable choices of $f$ and $m$.

EXAMPLE 3.6 (Parabolic surface $p$-Laplacian equation) Setting $w=u$ and $\mathcal{D}=\left|\nabla_{\Phi} u\right|^{p-2} \mathcal{I}$ for $p>1$ yields the following parabolic surface $p$-Laplacian equation:

$$
u_{t}=\nabla_{\Phi} \cdot\left(\left|\nabla_{\Phi} u\right|^{p-2} \nabla_{\Phi} u\right),
$$

which is the gradient flow for the energy

$$
E_{p}(u)=\frac{1}{p} \int_{\Omega}\left|\nabla_{\Phi} u\right|^{p}|\nabla \Phi| .
$$

EXAMPLE 3.7 (Cahn-Hilliard equation) Setting

$$
w=-\epsilon \Delta_{\Phi} u+\frac{1}{\epsilon} \psi^{\prime}(u),
$$

where $\psi$ is a double well potential (e.g. $\left.\psi(u)=\frac{1}{4}\left(u^{2}-1\right)^{2}\right)$, leads to the fourth order Cahn-Hilliard equation, [17],

$$
u_{t}=-\nabla_{\Phi} \cdot \mathcal{D} \nabla_{\Phi}\left(\epsilon \Delta_{\Phi} u-\frac{\psi^{\prime}(u)}{\epsilon}\right) .
$$

EXAMPLE 3.8 (Eulerian Allen-Cahn equation) Consideration of the $L^{2}$ gradient flow for the gradient energy functional

$$
E(v)=\int_{\Omega}\left(\frac{\epsilon}{2}\left|\nabla_{\Phi} v\right|^{2}+\frac{1}{\epsilon} \psi(v)|\nabla \Phi|\right)
$$

leads to

$$
\epsilon u_{t}=\epsilon \Delta_{\Phi} u-\frac{1}{\epsilon} \psi^{\prime}(u) .
$$




\subsection{Energy estimates}

3.3.1 Linear diffusion. A function $u \in H_{\Phi}^{1}(\Omega)$ is a weak solution of 3.8 if

$$
\int_{\Omega} u_{t} \eta|\nabla \Phi|+\int_{\Omega} \mathcal{D} \nabla_{\Phi} u \cdot \nabla_{\Phi} \eta|\nabla \Phi|=0
$$

for every $\eta \in H_{\Phi}^{1}(\Omega)$. Let $u$ be a weak solution. Then choosing $\eta=u$ leads to

$$
\frac{1}{2} \frac{\mathrm{d}}{\mathrm{d} t} \int_{\Omega} u^{2}|\nabla \Phi|+\int_{\Omega} \mathcal{D} \nabla_{\Phi} u \cdot \nabla_{\Phi} u|\nabla \Phi|=0
$$

and choosing $\eta=u_{t}$ leads to

$$
\int_{\Omega} u_{t}^{2}|\nabla \Phi|+\frac{1}{2} \frac{\mathrm{d}}{\mathrm{d} t} \int_{\Omega} \mathcal{D} \nabla_{\Phi} u \cdot \nabla_{\Phi} u|\nabla \Phi|=0 .
$$

3.3.2 Fourth order diffusion. Let $(u, w)$ be a weak solution. Then choosing $\eta=w$ and $\eta=u_{t}$ in the above equations 3.25, 3.26 leads to

$$
\begin{gathered}
\int_{\Omega} u_{t} w|\nabla \Phi|+\int_{\Omega} \mathcal{D} \nabla_{\Phi} w \cdot \nabla_{\Phi} w|\nabla \Phi|=0 \\
\frac{1}{2} \frac{\mathrm{d}}{\mathrm{d} t} \int_{\Omega}\left|\nabla_{\Phi} u\right|^{2}|\nabla \Phi|-\int_{\Omega} w u_{t}|\nabla \Phi|=0
\end{gathered}
$$

which yields

$$
\frac{1}{2} \frac{\mathrm{d}}{\mathrm{d} t} \int_{\Omega}\left|\nabla_{\Phi} u\right|^{2}|\nabla \Phi|+\int_{\Omega} \mathcal{D} \nabla_{\Phi} w \cdot \nabla_{\Phi} w|\nabla \Phi|=0
$$

3.3.3 Cahn-Hilliard equation. The pair $(u, w) \in\left(H_{\Phi}^{1}(\Omega)\right)^{2}$ is a weak solution of the surface Cahn-Hilliard equation if

$$
\begin{array}{r}
\int_{\Omega} u_{t} \eta|\nabla \Phi|+\int_{\Omega} \mathcal{D} \nabla_{\Phi} w \cdot \nabla_{\Phi} \eta|\nabla \Phi|=0 \\
\int_{\Omega}\left(\epsilon \nabla_{\Phi} u \cdot \nabla_{\Phi} \eta+\frac{1}{\epsilon} \psi^{\prime}(u) \eta\right)|\nabla \Phi|-\int_{\Omega} w \eta|\nabla \Phi|=0
\end{array}
$$

for all $\eta \in H_{\Phi}^{1}(\Omega)$. Let $(u, w)$ be a weak solution. Then choosing $\eta=w$ and $\eta=u_{t}$ in the above equations leads to

$$
\begin{array}{r}
\int_{\Omega} u_{t} w|\nabla \Phi|+\int_{\Omega} \mathcal{D} \nabla_{\Phi} w \cdot \nabla_{\Phi} w|\nabla \Phi|=0 \\
\frac{\mathrm{d}}{\mathrm{d} t} \int_{\Omega}\left(\frac{\epsilon}{2}\left|\nabla_{\Phi} u\right|^{2}+\frac{1}{\epsilon} \psi(u)\right)|\nabla \Phi|-\int_{\Omega} w u_{t}|\nabla \Phi|
\end{array}
$$

which yields

$$
\frac{\mathrm{d}}{\mathrm{d} t} \int_{\Omega}\left(\frac{\epsilon}{2}\left|\nabla_{\Phi} u\right|^{2}+\frac{1}{\epsilon} \psi(u)\right)|\nabla \Phi|+\int_{\Omega} \mathcal{D} \nabla_{\Phi} w \cdot \nabla_{\Phi} w|\nabla \Phi|=0 .
$$




\section{Finite element approximation}

\subsection{Semi-discrete approximation}

4.1.1 Linear diffusion. Our Eulerian SFEM is based on the the weak form 3.32). Find $U(\cdot, t) \in$ $S_{h}$ such that

$$
\int_{\Omega} U_{t} \eta|\nabla \Phi|+\int_{\Omega} \mathcal{D} \nabla_{\Phi} U \cdot \nabla_{\Phi} \eta|\nabla \Phi|=0 \quad \forall \eta \in S_{h}
$$

Setting

$$
U(\cdot, t)=\sum_{j=1}^{N} \alpha_{j}(t) \chi_{j}(\cdot)
$$

we find that

$$
\int_{\Omega} \sum_{j=1}^{N} \alpha_{j, t} \chi_{j} \eta|\nabla \Phi|+\int_{\Omega} \mathcal{D} \sum_{j=1}^{N} \alpha_{j}(t) \nabla_{\Phi} \chi_{j} \cdot \nabla_{\Phi} \eta|\nabla \Phi|=0 \quad \forall \eta \in S_{h}
$$

and taking $\eta=\chi_{k}, k=1, \ldots, N$, we obtain

$$
\mathcal{M} \dot{\alpha}+\mathcal{S} \alpha=0
$$

where $\mathcal{M}$ is the weighted mass matrix

$$
\mathcal{M}_{j k}=\int_{\Omega} \chi_{j} \chi_{k}|\nabla \Phi|, \quad j, k=1, \ldots, N
$$

and $\mathcal{S}$ is the weighted stiffness matrix

$$
\mathcal{S}_{j k}=\int_{\Omega} \mathcal{D} \nabla_{\Phi} \chi_{j} \nabla_{\Phi} \chi_{k}|\nabla \Phi|, \quad j, k=1, \ldots, N
$$

Because of the assumption on $\Phi$ the mass matrix $\mathcal{M}(t)$ is uniformly positive definite, so that we get existence and uniqueness of the semi-discrete finite element solution.

REMARK 4.1 A significant feature of our approach is the fact that the matrices $\mathcal{M}$ and $\mathcal{S}$ depend only on the evaluation of the gradient of the level set function $\Phi$. The method does not require an explicit numerical evaluation of surface quantities.

4.1.2 Fourth order linear diffusion. Find $(U(\cdot, t), W(\cdot, t)) \in\left(S_{h}\right)^{2}$ such that

$$
\begin{array}{r}
\int_{\Omega} U_{t} \eta|\nabla \Phi|+\int_{\Omega} \mathcal{D} \nabla_{\Phi} W \cdot \nabla_{\Phi} \eta|\nabla \Phi|=0, \\
\int_{\Omega} \nabla_{\Phi} U \cdot \nabla_{\Phi} \eta|\nabla \Phi|-\int_{\Omega} W \eta|\nabla \Phi|=0,
\end{array}
$$

for every $\eta \in S_{h}$. Setting

$$
U(\cdot, t)=\sum_{j=1}^{N} \alpha_{j}(t) \chi_{j}(\cdot), \quad W(\cdot, t)=\sum_{j=1}^{N} \beta_{j}(t) \chi_{j}(\cdot)
$$


we find that, for all $\eta \in S_{h}$,

$$
\begin{array}{r}
\int_{\Omega} \sum_{j=1}^{N} \alpha_{j, t} \chi_{j} \eta|\nabla \Phi|+\int_{\Omega} \mathcal{D} \sum_{j=1}^{N} \beta_{j}(t) \nabla_{\Phi} \chi_{j} \cdot \nabla_{\Phi} \eta|\nabla \Phi|=0 \\
\int_{\Omega} \sum_{j=1}^{N} \alpha_{j}(t) \nabla_{\Phi} \chi_{j} \cdot \nabla_{\Phi} \eta|\nabla \Phi|-\int_{\Omega} \sum_{j=1}^{N} \beta_{j} \chi_{j} \eta|\nabla \Phi|=0,
\end{array}
$$

and taking $\eta=\chi_{k}, k=1, \ldots, N$, we obtain

$$
\begin{aligned}
\mathcal{M} \dot{\alpha}+\mathcal{S} \beta & =0 \\
\mathcal{S}^{0} \alpha-\mathcal{M} \beta & =0
\end{aligned}
$$

where

$$
\mathcal{S}_{j k}^{0}=\int_{\Omega} \nabla_{\Phi} \chi_{j} \nabla_{\Phi} \chi_{k}|\nabla \Phi|, \quad j, k=1, \ldots, N
$$

which yields

$$
\mathcal{M} \dot{\alpha}+\mathcal{S M}^{-1} \mathcal{S}^{0} \alpha=0
$$

4.1.3 Cahn-Hilliard equation. We formulate a mixed finite element scheme based on the splitting into second order elliptic operators (cf. [7]) to obtain the scheme: find $(U(\cdot, t), W(\cdot, t)) \in$ $\left(S_{h}\right)^{2}$ such that

$$
\begin{array}{r}
\int_{\Omega} U_{t} \eta|\nabla \Phi|+\int_{\Omega} \mathcal{D} \nabla_{\Phi} W \cdot \nabla_{\Phi} \eta|\nabla \Phi|=0, \\
\epsilon \int_{\Omega} \nabla_{\Phi} U \cdot \nabla_{\Phi} \eta|\nabla \Phi|+\int_{\Omega} \frac{1}{\epsilon} \Pi_{h}\left(\psi^{\prime}(U)\right) \eta|\nabla \Phi|-\int_{\Omega} W \eta|\nabla \Phi|=0,
\end{array}
$$

for every $\eta \in S_{h}$. Here we use $\Pi_{h}$ to denote the usual interpolation operator for $S_{h}$. Setting

$$
U(\cdot, t)=\sum_{j=1}^{N} \alpha_{j}(t) \chi_{j}(\cdot), \quad W(\cdot, t)=\sum_{j=1}^{N} \beta_{j}(t) \chi_{j}(\cdot),
$$

in a similar manner to the fourth order linear diffusion case we find that

$$
\begin{aligned}
\mathcal{M} \dot{\alpha}+\mathcal{S} \beta & =0, \\
\epsilon \mathcal{S}^{0} \alpha+\frac{1}{\epsilon} \mathcal{M} \Psi^{\prime}(\alpha)-\mathcal{M} \beta & =0,
\end{aligned}
$$

where $\left\{\Psi^{\prime}(\alpha)\right\}_{j}=\psi^{\prime}\left(\alpha_{j}\right)$, which yields

$$
\mathcal{M} \dot{\alpha}+\epsilon \mathcal{S} \mathcal{M}^{-1} \mathcal{S}^{0} \alpha+\frac{1}{\epsilon} \mathcal{S} \Psi^{\prime}(\alpha)=0 .
$$




\subsection{Time stepping}

4.2.1 Implicit Euler scheme. The time discretization in our computations may be carried out by an implicit method. We introduce a time step size $\tau>0$ and use upper indices for the time levels. Thus $U^{m}$ represents $U(\cdot, m \tau)$. With these notations we propose the following algorithm.

Algorithm 4.2 (Fully discrete scheme) Let $U^{0} \in S_{h}$ be given. For $m=0, \ldots, m_{T}$ solve the linear system

$$
\frac{1}{\tau} \int_{\Omega} U^{m+1} \chi_{j}|\nabla \Phi|+\int_{\Omega} \mathcal{D} \nabla_{\Phi} W^{m+1} \cdot \nabla_{\Phi} \chi_{j}|\nabla \Phi|=\frac{1}{\tau} \int_{\Omega} U^{m} \chi_{j}|\nabla \Phi|
$$

for all $j=1, \ldots, N$.

In the case of the second order linear diffusion equation $W=U$ this leads to the linear algebraic system

$$
(\mathcal{M}+\tau \mathcal{S}) \alpha^{m+1}=\mathcal{M} \alpha^{m}
$$

For the linear fourth order diffusion equation with $\mathcal{D}=I$ we obtain the linear system

$$
\left(\mathcal{M}+\tau \mathcal{S}^{0} \mathcal{M}^{-1} \mathcal{S}^{0}\right) \alpha^{m+1}=\mathcal{M} \alpha^{m}
$$

Gradient stable time stepping schemes for the Cahn-Hilliard system such as backward Euler and convexity splitting are formulated, for example, in [4, 16]. Here we chose to implement the following simple scheme with $\mathcal{D}=I$ :

$$
\left(\mathcal{M}+\tau \epsilon \mathcal{S}^{0} \mathcal{M}^{-1} \mathcal{S}^{0}\right) \alpha^{m+1}=\mathcal{M} \alpha^{m}-\frac{1}{\epsilon} \mathcal{S} \Psi^{\prime}\left(\alpha^{m}\right) .
$$

The symmetric systems in the computational examples are solved using the conjugate gradient algorithm which was adequate for the purposes of this work.

\section{Numerical results}

EXAMPLE 5.1 To start, we solve the heat equation on a circle. We choose $\Omega$ to be the annular region with outer radius 1 and inner radius 0.5 . We set $\Phi(x)=|x|-0.75$ so that the boundary $\partial \Omega$ comprises level lines of $\Phi$. The function $u(x, t)=\exp \left(-t /|x|^{2}\right) x_{2} /|x|$ is an exact solution of

$$
u_{t}-\Delta_{\Gamma} u=0
$$

on $\Gamma(t)=\Gamma_{0}=\left\{x \in \mathbb{R}^{2}:|x|=0.5\right\}$ with initial data $u_{0}(x)=x_{2} /|x|$. We have chosen the coupling $\tau=h^{2}$ in order to show the higher order convergence for $L^{2}$ and $L^{\infty}$ errors. The time interval is $T=1.0$. In Table 1 we show the absolute errors and the corresponding experimental orders of convergence for the norms on $\Omega$,

$$
\begin{aligned}
& L^{\infty}\left(L_{\Phi}^{2}(\Omega)\right)=\sup _{(0, T)}\left\|u-u_{h}\right\|_{L_{\Phi}^{2}(\Omega)}, \quad L^{2}\left(H_{\Phi}^{1}(\Omega)\right)=\left(\int_{0}^{T}\left\|\nabla_{\Phi}\left(u-u_{h}\right)\right\|_{L_{\Phi}^{2}(\Omega)}^{2}\right)^{1 / 2}, \\
& L^{\infty}\left(L^{\infty}(\Omega)\right)=\sup _{(0, T)}\left\|u-u_{h}\right\|_{L^{\infty}(\Omega)}, \quad L^{2}\left(H^{1}(\Omega)\right)=\left(\int_{0}^{T}\left\|\nabla\left(u-u_{h}\right)\right\|_{L^{2}(\Omega)}^{2}\right)^{1 / 2},
\end{aligned}
$$


and for the following norms on the zero level set $\Gamma_{0}=\left\{x \in \mathbb{R}^{2}: \Phi(x)=0\right\}$ :

$$
\begin{aligned}
& L^{\infty}\left(L_{\Phi}^{2}\left(\Gamma_{0}\right)\right)=\sup _{(0, T)}\left\|u-u_{h}\right\|_{L_{\Phi}^{2}\left(\Gamma_{0}\right)}, \quad L^{2}\left(H_{\Phi}^{1}\left(\Gamma_{0}\right)\right)=\left(\int_{0}^{T}\left\|\nabla_{\Phi}\left(u-u_{h}\right)\right\|_{L_{\Phi}^{2}\left(\Gamma_{0}\right)}^{2}\right)^{1 / 2}, \\
& L^{\infty}\left(L^{\infty}\left(\Gamma_{0}\right)\right)=\sup _{(0, T)}\left\|u-u_{h}\right\|_{L^{\infty}\left(\Gamma_{0}\right)} .
\end{aligned}
$$

For an error $E\left(h_{1}\right)$ and $E\left(h_{2}\right)$ for the grid sizes $h_{1}$ and $h_{2}$ the experimental order of convergence is defined as $\operatorname{eoc}\left(h_{1}, h_{2}\right)=\log \frac{E\left(h_{1}\right)}{E\left(h_{2}\right)}\left(\log \frac{h_{1}}{h_{2}}\right)^{-1}$.

TABLE 1

Heat equation on circles. Errors and experimental orders of convergence for Example 5.1

\begin{tabular}{l|ll|ll|ll|ll}
\hline$h$ & $L^{\infty}\left(L_{\Phi}^{2}(\Omega)\right)$ & eoc & $L^{2}\left(H_{\Phi}^{1}(\Omega)\right)$ & eoc & $L^{2}\left(H^{1}(\Omega)\right)$ & eoc & $L^{\infty}\left(L^{\infty}(\Omega)\right)$ & eoc \\
\hline 0.5176 & 0.07401 & - & 0.1090 & - & 0.09565 & - & 0.1139 & - \\
0.2831 & 0.02594 & 1.74 & 0.03986 & 1.67 & 0.1325 & 0.93 & 0.04539 & 1.52 \\
0.1500 & 0.007796 & 1.89 & 0.01587 & 1.45 & 0.07188 & 0.96 & 0.01696 & 1.55 \\
0.07716 & 0.002192 & 1.91 & 0.007147 & 1.20 & 0.03879 & 0.93 & 0.006144 & 1.53 \\
0.03912 & 0.0006067 & 1.89 & 0.003438 & 1.08 & 0.02042 & 0.95 & 0.002333 & 1.43 \\
0.01969 & 0.0001694 & 1.86 & 0.001699 & 1.03 & 0.01061 & 0.95 & 0.0009357 & 1.33 \\
\hline
\end{tabular}

\begin{tabular}{l|ll|ll|ll}
\hline$h$ & $L^{\infty}\left(L_{\Phi}^{2}\left(\Gamma_{0}\right)\right)$ & eoc & $L^{2}\left(H_{\Phi}^{1}\left(\Gamma_{0}\right)\right)$ & eoc & $L^{\infty}\left(L^{\infty}\left(\Gamma_{0}\right)\right)$ & eoc \\
\hline 0.5176 & 0.07401 & - & 0.0874 & - & 0.06148 & - \\
0.2831 & 0.03142 & 1.77 & 0.04512 & 1.10 & 0.02389 & 1.57 \\
0.1500 & 0.009560 & 1.88 & 0.02653 & 0.84 & 0.009116 & 1.52 \\
0.07716 & 0.002690 & 1.91 & 0.01149 & 1.26 & 0.002446 & 1.98 \\
0.03912 & 0.0006343 & 2.13 & 0.006237 & 0.90 & 0.0005447 & 2.21 \\
0.01969 & 0.0001484 & 1.89 & 0.002943 & 1.10 & 0.0001484 & 1.89 \\
\hline
\end{tabular}

EXAMPLE 5.2 In Figure 1 we show a computation on the domain $\Omega=(-1,1) \times(-1,1)$ of the solution of

$$
u_{t}-\Delta_{\Phi} u=0
$$

with $\Phi(x)=x_{2}$ and initial value $u_{0}=\chi_{\Omega_{0}}$, where $\Omega_{0}=(-1,-0.9) \times(-0.5,0.5)$. The grid orientation is $(1,-1),(0,1),(1,0),(1,1)$ and thus aligned with the levels of $\Phi$. The grid size was $h=0.04419$ and time step size was $\tau=0.1 h^{2}$. We see that the numerical diffusion vertical to the levels of $\Phi$ is quite small. The figure shows the level lines of the solution between $u=0.05$ and $u=1.0$ and the distance of the level lines is 0.05 .

In Figure 2 we show a similar example but now we have distributed the "initial mass" on $\Omega_{0}=$ $\left\{\left(x_{1}, x_{2}\right):-1<x_{1}<\delta, x_{1}-\delta-\frac{1}{2} \sqrt{3}<\sqrt{3} x_{2}<x_{1}-\delta+\frac{1}{2} \sqrt{3}\right\}$. With the choice $\delta=0.8997889$ the same amount of mass has to be diffused along the levels of $\Phi$ as in the previous example. We have now chosen $\Phi(x)=0.5\left(x_{1}-\sqrt{3} x_{2}\right)$ so that the levels of $\Phi$ are not aligned with the grid anymore. We observe that the numerical diffusion vertical to the levels of $\Phi$ appears to be larger than for the previous orientation of the levels of $\Phi$. The level lines in Figure 2 are spaced as in the previous figure. 

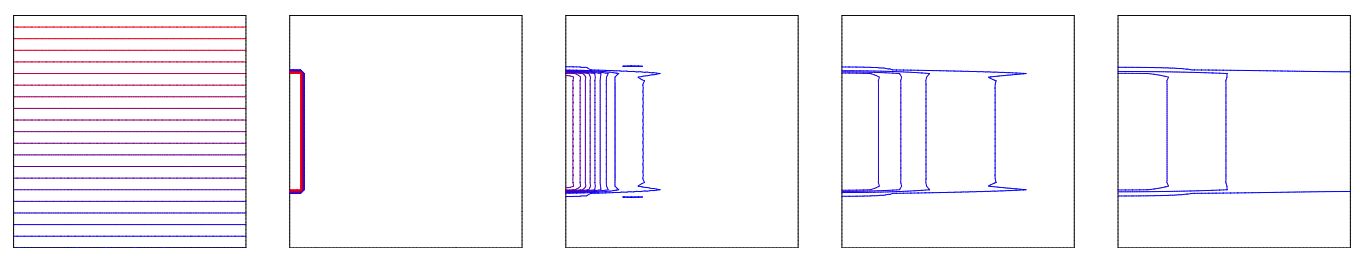

FIG. 1. Example 5.2 Horizontal level lines of $\Phi$ (left). Level lines of the solution at time steps 0, 100, 500, 1300.
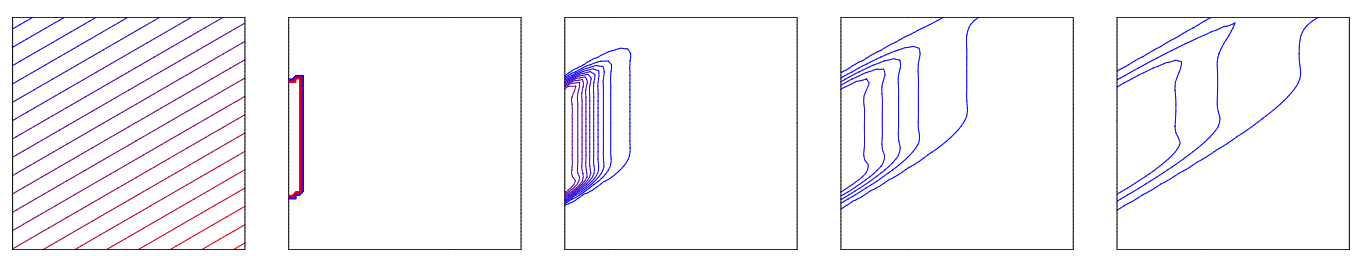

FIG. 2. Example 5.2 Level lines of $\Phi$ (left). Level lines of the solution at time steps 0, 100, 500, 1300.

EXAMPLE 5.3 Additionally we computed the same situation as in the first part of Example 5.2 but with initial function $u_{0}=0$ and with the right hand side $f=100 \sin \left(20 \pi x_{2}\right) \chi_{(-1,-0.9) \times(-1,1)}$. The results are shown in Figure 3 .
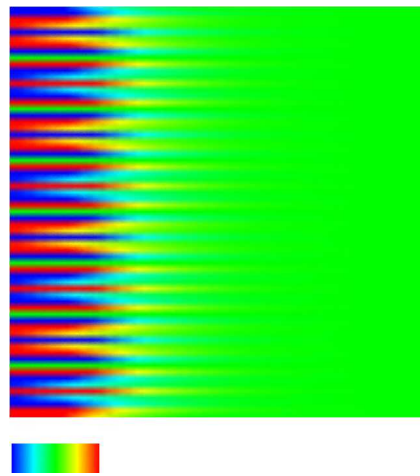

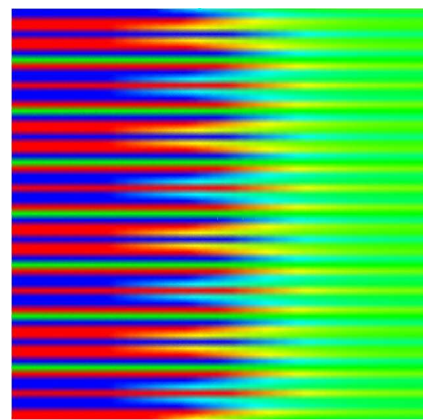

101

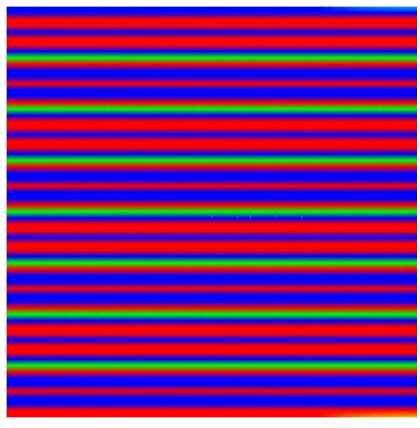

101

FIG. 3. Values between -1 and 1 of the solution of Example 5.3 for the time steps 50, 100 and 450 . The colour scale is shown. We only show values of the solution between -1 and 1 . Blue represents the value -1 , green 0.0 and red the value 1 . (For the colours here and in subsequent figures, see the pdf file at http://www.ems-ph.org.)

EXAMPLE 5.4 In the case of homogeneous Neumann boundary conditions the solution is conserved on each level surface. Thus we expect that the solution $u$ evolves to a stationary solution which is constant on each level line of $\Phi$. In this example we take the level set function $\Phi(x)=x_{2}-2\left(1-x_{2}^{2}\right) \sin (0.3) \sin \left(2 \pi x_{1}\right)$ and the initital value $u_{0}\left(x_{1}, x_{2}\right)=x_{2}$ on the domain $\Omega=(-1,1) \times(-1,1)$. In Figure 4 we show level lines of $u$ for several time steps. The function $u$ becomes constant on the level lines of $\Phi$. Therefore in Figure 4 we do not plot the levels of $\Phi$, since they are nearly identical with the last shown time step. 

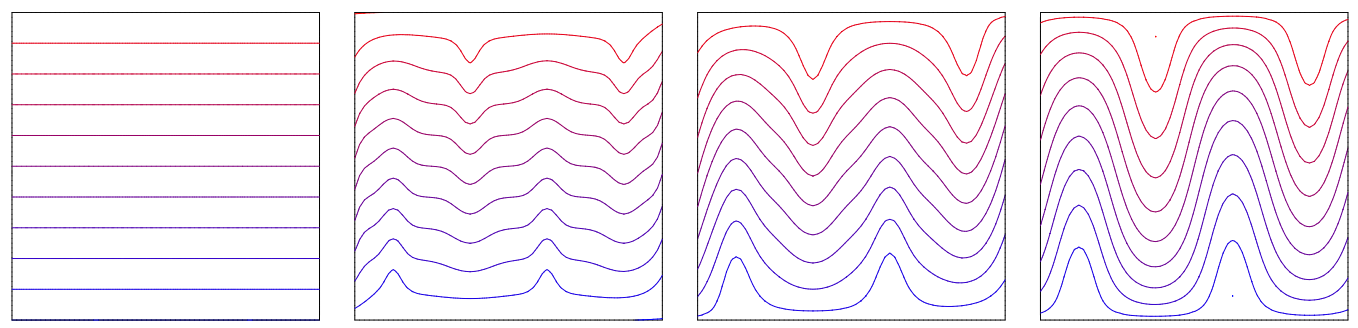

FIG. 4. Level lines of the solution $u$ for the time steps $0,100,400$ and 5200. The last picture nearly shows the level lines of $\Phi$. Levels between -1 and 1 equally spaced with increment 0.2 are shown.

EXAMPLE 5.5 Let now $\Omega$ be the unit disk. We solve with initial data $u_{0}(x)=x_{1}$ on the levels of the level set function $\Phi\left(x_{1}, x_{2}, t\right)=\left(x_{1}-0.25\right)^{2}+\left(x_{2}+0.1\right)^{2}$, so that the level sets of $\Phi$ are circles. (Note the degeneracy of $\Phi$ at the centre). In this example we solve under Dirichlet boundary conditions $u(\cdot, t)=u_{0}$ on $\partial \Omega$. Thus we see that $\Omega$ may be decomposed into sets $\Omega_{2}$ in which the level lines have two end points on $\partial \Omega, \Omega_{1}$ with no end points on $\partial \Omega$ and a dividing single level line $\Gamma_{*}$ which touches $\partial \Omega$ at just one point. Clearly the Dirichlet boundary condition plays a role in the evolution on the level surfaces in $\Omega_{2}$ and in the long time the solution converges to a solution of a boundary value problem which is linear in the arc length on each circular level surface. On the other hand, the solution is conserved on closed level surfaces and in the long time the solution converges to a constant on these circles. This may be observed in the figures. We used a triangulation with 1089 nodes, $h=0.1137$ and $\tau=0.001294$.
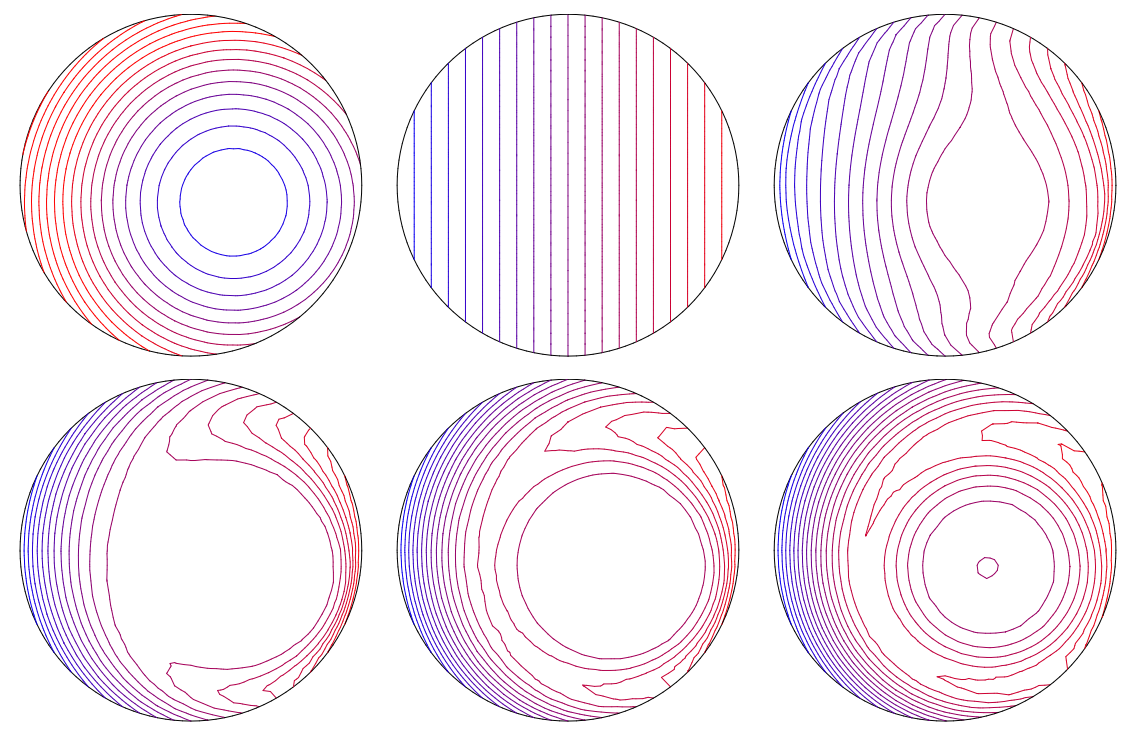

FIG. 5. Solution of the $\Phi$-heat equation under Dirichlet boundary conditions. Levels of $\Phi$ and then levels of $u$ for the time steps $0,200,800,1500$ and 3000. Levels between -1 and 1 are shown at an increment of 0.1 . 
EXAMPLE 5.6 We show computations for a three-dimensional problem with homogeneous Neumann boundary conditions. The computational domain is $\Omega=(-1,1)^{3}$, and we use the level set function

$$
\Phi\left(x_{1}, x_{2}, x_{3}\right)=x_{1} x_{3}-2\left(1-x_{3}^{2}\right) \sin 0.3 \sin \left(2 \pi x_{1}\right) .
$$

The initial value is taken to be $u_{0}\left(x_{1}, x_{2}, x_{3}\right)=x_{2}$. In Figures 6 , 7 and 8 we show various level surfaces of $\Phi$ which are coloured according to the values of $u_{h}$ at three time steps. The continuous solution tends to a constant on the levels of $\Phi$. The time step size was $\tau=3.1250 e-04$ and we used piecewise linear elements with 35937 nodes. The colour coding is such that blue corresponds to the value -1 , red to the value 1 with a linear scale between.

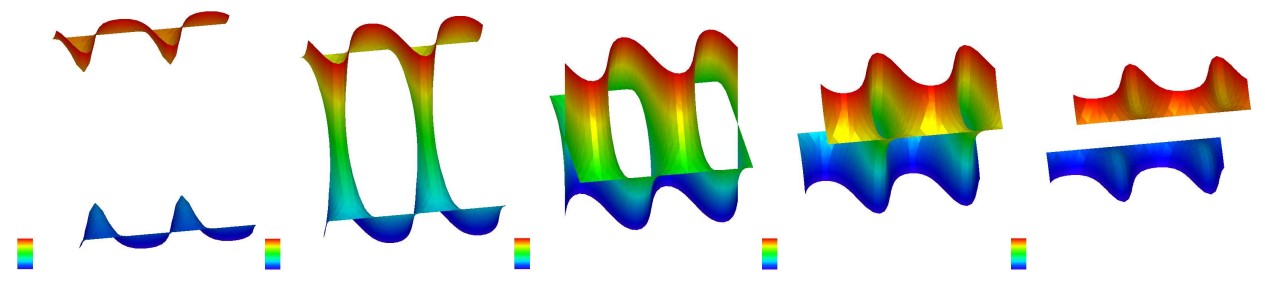

FIG. 6. Level surfaces $\Phi=-0.75,-0.5,0.0,0.5,0.75$ with colouring according to the values of $u_{h}(\cdot, 0)$.

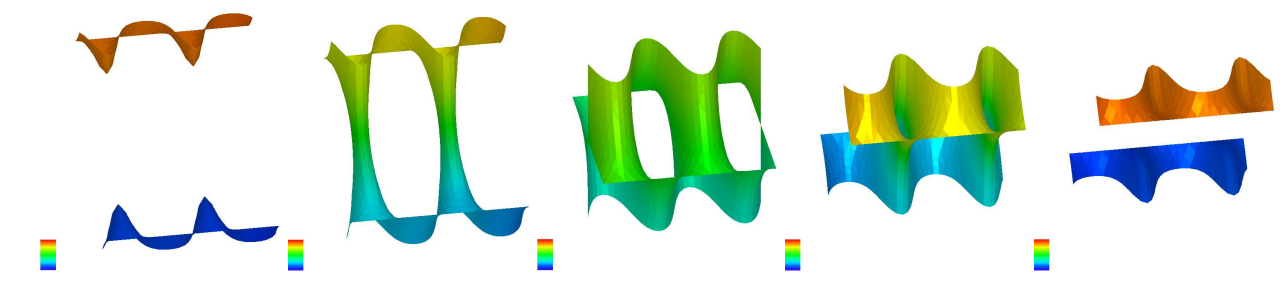

FIG. 7. Level surfaces $\Phi=-0.75,-0.5,0.0,0.5,0.75$ with colouring according to the values of $u_{h}: 1000$-th time step.

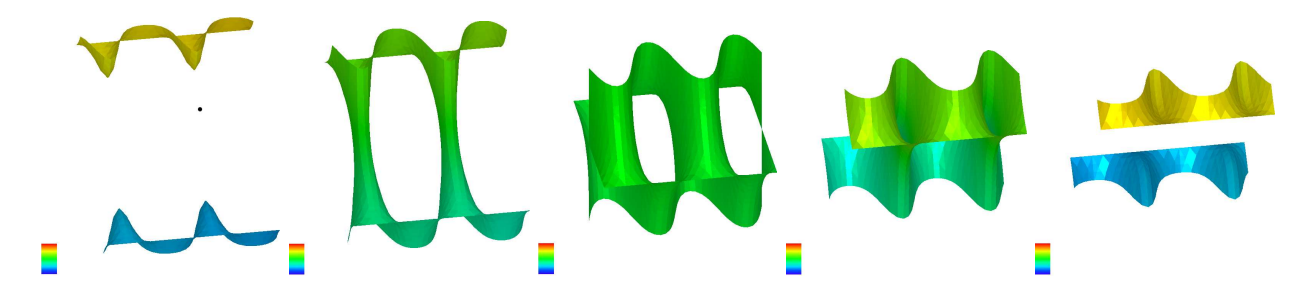

FIG. 8. Level surfaces $\Phi=-0.75,-0.5,0.0,0.5,0.75$ with colouring according to the values of $u_{h}: 3900$-th time step.

EXAMPLE 5.7 The purpose of this computational example is to show how our algorithm behaves for a nonsmooth level set function $\Phi$. Thus this result is purely experimental. We have chosen $\Omega=(-1,1) \times(-1,1)$ as domain and

$$
\Phi\left(x_{1}, x_{2}\right)=\sqrt{\left(x_{1}-x_{01}\right)^{2}+\delta^{2}}+\sqrt{\left(x_{2}-x_{02}\right)^{2}+\delta^{2}}
$$

as a regularization of $\Phi\left(x_{1}, x_{2}\right)=\left|x_{1}-x_{01}\right|+\left|x_{2}-x_{02}\right|$ with $x_{01}=0.25, x_{02}=-0.75$ and $\delta=10^{-4}$. As initial value we have taken the function $u_{0}\left(x_{1}, x_{2}\right)=x_{1}$. In Figure 9 we show 
the level lines of $\Phi$ and the colouring is done according to the values of the solution $u$. We have used the grid size $h=0.044$ and the time step size $\tau=0.0002$. Note that we are approximating solutions on curves with corners. Computationally there were no special problems in connection with the "nonsmoothness" of $\Phi$ or with the size of the regularization parameter $\delta$. Again we consider homogeneous Neumann boundary conditions so that the solution is conserved on each level set. Since the level curves have different lengths and in each corner of $\Omega$ there is a level line which divides the level lines into a set without corners and a set with corners. The length of the level lines varies jumps discontinuously at these dividing lines. Thus in the steady state we expect the development of a solution which jumps discontinuously across these dividing lines. This is seen clearly in the third image of Figure 9 and in Figure 10
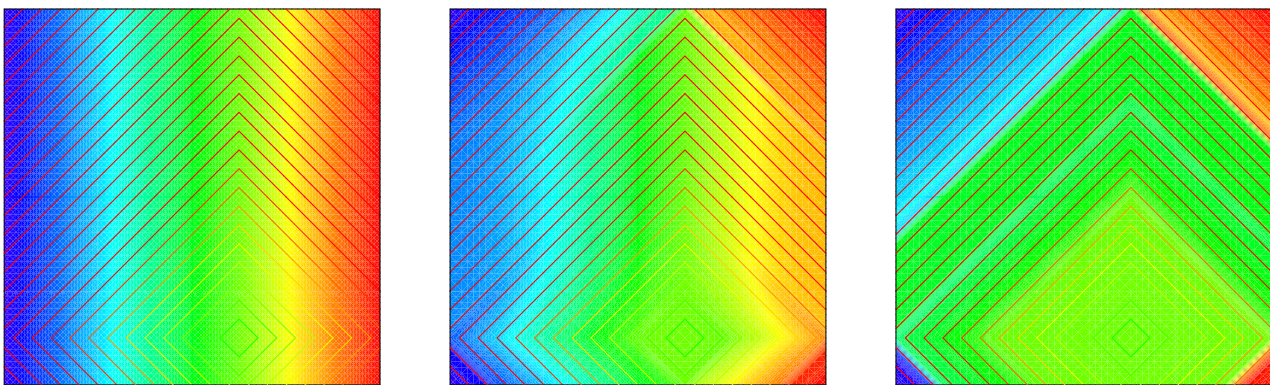

FIG. 9. Level lines of $\Phi$ (left) and level lines of the solution $u$ for the time steps $t=0.00, t=0.1953$ and $t=3.164$. The colouring of the values of u ranges between blue $(-1.0)$ and red (1.0). We also show the levels of $\Phi$.

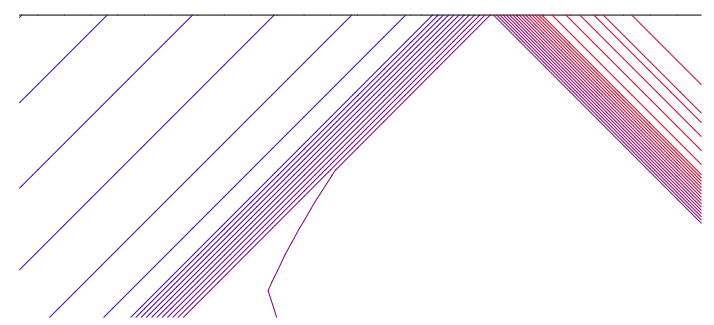

FIG. 10. Equally spaced level lines of the solution at time 3.164 close to the middle of the upper part of the domain $\Omega$ exhibiting the development of a discontinuity of $u$.

EXAMPLE 5.8 We solve the Cahn-Hilliard equation on the annulus with inner radius 0.5 and outer radius 1.0. The levels of $\Phi$ are concentric circles,

$$
\Phi(x)=|x|-0.75
$$

and as initial function we choose

$$
u_{0}(x)=0.01 \sin \left(4 \varphi+r_{1}\right)\left(\sin \left(7 \varphi+r_{2}\right)-0.25\right) \sin \left(10 \varphi+r_{3}\right),
$$

where $r_{1}, r_{2}$ and $r_{3}$ are random numbers from $(0,1)$ and $\varphi$ is the polar angle in the $x_{1}, x_{2}$-plane. The parameter $\epsilon$ was chosen as 0.05 . The grid size was $h=0.02492$ and the time step size was $\tau=0.0006215$. The results are shown in Figure 11 

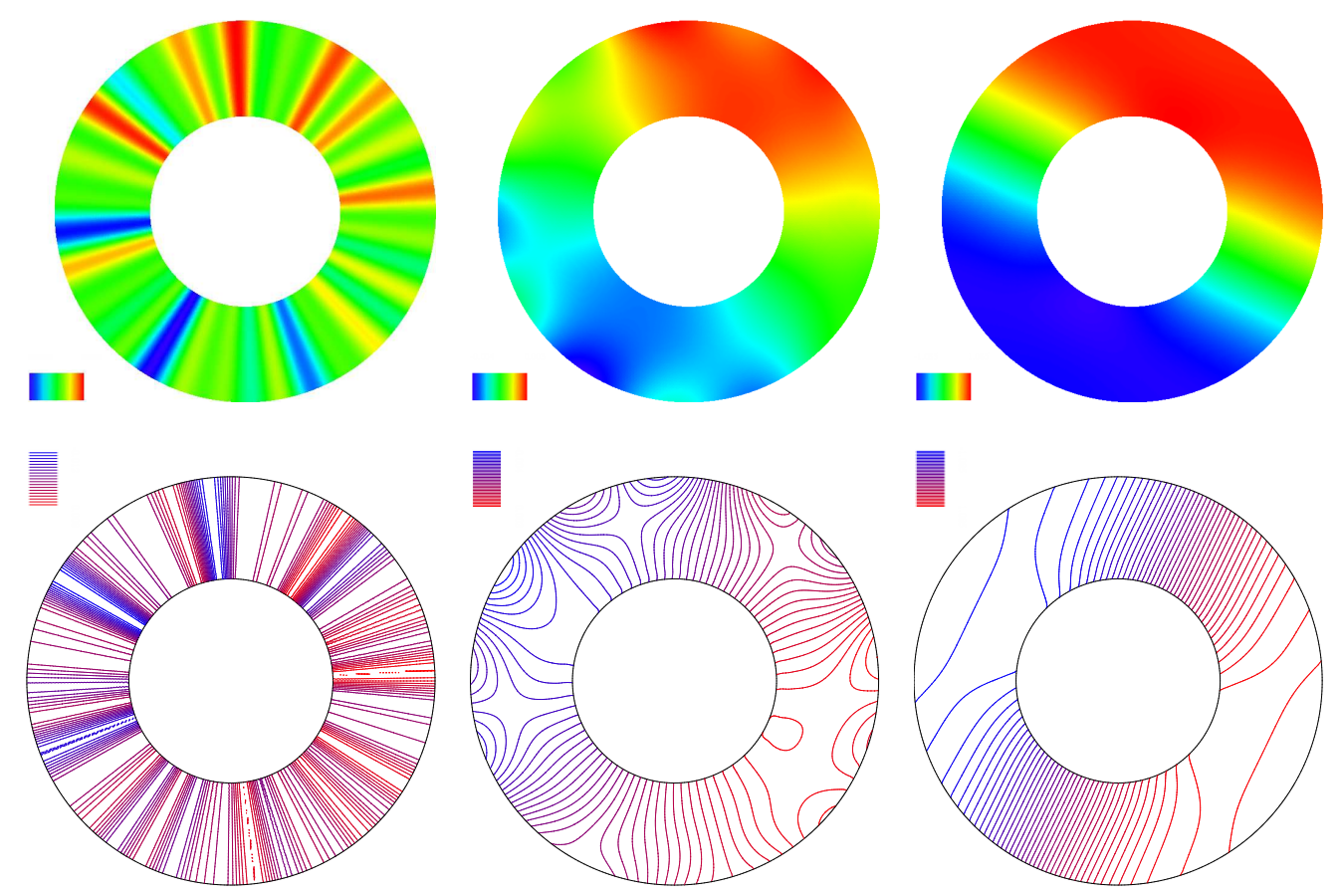

FIG. 11. Solution of the $\Phi$-Cahn-Hilliard equation on an annulus with $\Phi$ as in 5.1. From left to right we show the solution at the times $t=0.0, t=0.006837$ and $t=0.7402$. The values are coloured between minimum and maximum of the solution according to the scale shown. Second row: plot of the level lines of the solution for the same situations.

In order to test the numerical diffusion in the direction vertical to the levels of $\Phi$ we compute the following example. The domain $\Omega$ and $\Phi$ are chosen as in the previous example. We choose as initial value the discontinuous function

$$
u_{0}(x)=\chi\{x: \| x|-0.75|<0.01\}(x) 0.1 \sin (20 \pi \varphi) .
$$

The results in Figure 12 show that the decomposition effect appears to be quite local on $|x|=0.75$.

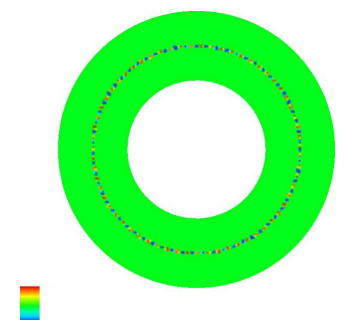

च

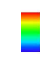

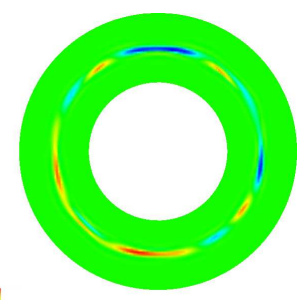

]

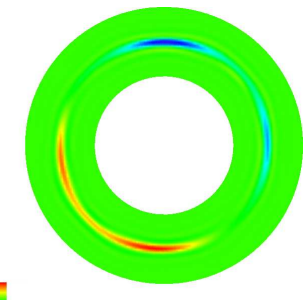

=

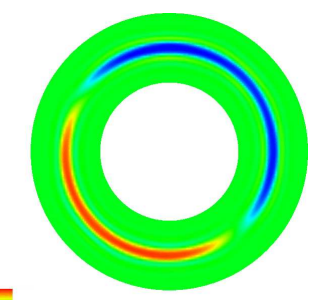

플

FIG. 12. Solution of the $\Phi$-Cahn-Hilliard equation on an annulus with $\Phi$ as in 5.1 and the initial value 5.2. From left to right we show level lines of the solution at the times $t=0.0, t=0.002386, t=0.04360$ and $t=0.1304$. The values are coloured between minimum (blue) and maximum (red) of the solution according to the scale shown in Figure 11 Note that the green colour stands for values of the solution close to 0 . 


\section{Concluding remarks}

We have shown how the finite element method can be employed to solve PDEs on implicit surfaces. A numerical example indicates that the rate of convergence is optimal. In a practical example where one wishes to use this approach to approximate the solution on just one surface there are some issues to be addressed. In particular we mention the need to extend the data off the given surface $\Gamma_{0}$ into a neighbourhood and to consider the formulation in a narrow band. These have been addressed in the context of finite difference approximations in [19, 20]. An $h$-narrow band formulation of our finite element approach to equations on implicit surfaces is proposed in [8] for elliptic equations. An optimal order $H^{1}(\Gamma)$ error bound is proved and numerical experiments are presented which indicate higher order convergence in $L^{2}(\Gamma)$. The numerical analysis in the case of parabolic equations and computations on large domains $\Omega$ is still open.

In [14] we have extended the method proposed in this paper to parabolic equations on evolving surfaces in the spirit of our evolving surface finite element method (ESFEM). An aim is to develop a numerical tool for the computation of the motion of an interface with complex morphology on which a concentration is being diffused and advected (see for example [15]).

\section{Acknowledgements}

This work was began whilst the authors participated in the 2003 programme Computational Challenges in Partial Differential Equations at the Isaac Newton Institute, Cambridge, UK. The work was supported by the Deutsche Forschungsgemeinschaft via DFG-Forschergruppe Nonlinear Partial Differential Equations: Theoretical and Numerical Analysis and by the UK EPSRC via the Mathematics Research Network: Computation and Numerical Analysis for Multiscale and Multiphysics Modelling.

Part of this work was done during a stay of the first author at the ICM at the University of Warsaw supported by the Alexander von Humboldt Honorary Fellowship 2005 granted by the Foundation for Polish Science.

The three-dimensional computations were done with the program ALBERTA [26] and the graphical presentations were performed with the package GRAPE. We specially thank Martin Lenz and Robert Klöfkorn for help with GRAPE.

\section{REFERENCES}

1. Adalsteinsson, D., \& Sethian, J. A. Transport and diffusion of material quantities on propagating interfaces via level set methods. J. Comput. Phys. 185 (2003), 271-288. Zbl 1047.76093 MR 2010161

2. Aubin, Th., Nonlinear Analysis on Manifolds. Monge-Ampère Equations. Springer, Berlin (1982). Zbl 0512.53044 MR 0681859

3. Bertalmio, M., Cheng, L. T., Osher, S., \& Sapiro, G. Variational problems and partial differential equations on implicit surfaces. J. Comput. Phys. 174 (2001), 759-780. Zbl 0991.65055 MR 1868103

4. Blowey, J. F., \& Elliott, C. M. The Cahn-Hilliard gradient theory for phase separation with nonsmooth free energy. Part II: Numerical Analysis. Eur. J. Appl. Math. 3 (1992), 147-179. Zbl 0810.35158 MR 1166255

5. BURGER, M., Finite element approximation of elliptic partial differential equations on implicit surfaces. Comput. Visual. Sci. (2007), to appear.

6. Cheng, L., Burchard, P., Merriman, B., \& Osher, S. Motion of curves constrained on surfaces using a level set approach. J. Comput. Phys. 175 (2002), 604-644. Zbl 0996.65013 MR 1880120

7. Copetti, M., \& Elliott, C. M. Numerical analysis of the Cahn-Hilliard equation with a logarithmic free energy. Numer. Math. 63 (1992), 39-65. Zbl 0762.65074 MR 1182511 
8. Deckelnick, K. P., Dziuk, G., Elliott, C. M., \& Heine, C. J. An $h$-narrow band finite element method for elliptic equations on implicit surfaces. In preparation.

9. Deckelnick, K., Elliott, C. M., \& Styles, V. Numerical diffusion induced grain boundary motion. Interfaces Free Bound. 3 (2001), 393-414. Zbl 0991.35095 MR 1869586

10. Dogel, J., Tsekov, R., \& Freyland, W. Two dimensional connective nano-structures of electrodeposited $\mathrm{Zn}$ on $\mathrm{Au}$ (111) induced by spinodal decomposition. J. Chemical Phys. 122 (2005), 094703, 8 pp.

11. DZIUK, G., Finite elements for the Beltrami operator on arbitrary surfaces. Partial Differential Equations and Calculus of Variations, S. Hildebrandt and R. Leis (eds.), Lecture Notes in Math. 1357, Springer, Berlin (1988), 142-155. Zbl 0663.65114 MR 0976234

12. Dziuk, G., \& Elliott, C. M. Finite elements on evolving surfaces. IMA J. Numer. Anal. 27 (2007), 262-292. Zbl 1120.65102 MR 2317005

13. Dziuk, G., \& Elliott, C. M. Surface finite elements for parabolic equations. J. Comput. Math. 25 (2007), 385-407. MR 2337402

14. Dziuk, G., \& Elliott, C. M. Eulerian level set method for PDEs on evolving surfaces. Comput. Visual. Sci., to appear.

15. Eilks, C., \& Elliott, C. M. Numerical simulation of dealloying by surface dissolution via the evolving surface finite element method. In preparation.

16. Elliott, C. M., The Cahn-Hilliard model for the kinetics of phase separation. Mathematical Models for Phase Change Problems, J. F. Rodrigues (ed.), Int. Ser. Numer. Math. 88, Birkhäuser (1989), 35-73. Zbl 0692.73003 MR 1038064

17. Elliott, C. M., \& Zheng, S. M. On the Cahn-Hilliard equation. Arch. Ration. Mech. Anal. 96 (1986), 339-357. Zbl 0624.35048 MR 0855754

18. Gilbarg, D., \& Trudinger, N. S. Elliptic Partial Differential Equations of Second Order. Springer (1988).

19. Greer, J. B., An improvement of a recent Eulerian method for solving PDEs on general geometries. J. Sci. Comput. 29 (2006), 321-352. Zbl 1122.65073 MR 2272322

20. Greer, J., Bertozzi, A., \& S APIRo, G. Fourth order partial differential equations on general geometries. J. Comput. Phys. 216 (2006), 216-246. Zbl 1097.65087 MR 2223442

21. Halpern, D., Jensen, O. E., \& Grotberg, J. B. A theoretical study of surfactant and liquid delivery into the lung. J. Appl. Physiology 85 (1998), 333-352.

22. Kimmel, R., Intrinsic scale space for images on surfaces: The geodesic curvature flow. Graph. Models Image Process. 59 (1997), 365-372.

23. Memoli, F., SAPiro, G., \& Thompson, P. Implicit brain imaging. NeuroImage 23 (2004), S179-S188.

24. Myers, T. G., Charpin, J. P. F., \& Chapman, S. J. The flow and solidification of a thin fluid film on an arbitrary three dimensional surface. Phys. Fluids 14 (2002), 2788-2803. MR 1917313

25. Ratz, A., \& Voigt, A. PDEs on surfaces-a diffuse interface approach. Comm. Math. Sci. 4 (2006), 575-590. MR 2247931

26. Schmidt, A., \& Siebert, K. G. Design of Adaptive Finite Element Software: The Finite Element Toolbox ALBERTA. Lecture Notes in Comput. Sci. Engrg. 42, Springer (2005). Zbl 1068.65138 MR 2127659

27. Schönborn, O., \& Desai, R. C. Kinetics of phase ordering on curved surfaces. Phys. A 239 (1997), 412-419.

28. SPIRA, A., \& Kimmel, R. Geometric curve flows on parametric manifolds. J. Comput. Phys. 223 (2007), 235-249. Zbl 1118.53004 MR 2314390

29. TANG, P., QIU, F., ZhANG, H. D., \& YANG, Y. L. Phase separation patterns for diblock copolymers on spherical surfaces: A finite volume method. Phys. Rev. E 72 (2005), 016710, 7 pp.

30. XU, J.-J., \& ZHAO, H.-K. An Eulerian formulation for solving partial differential equations along a moving interface. J. Sci. Comput. 19 (2003), 573-594. Zbl 1081.76579 MR 2028859 